\title{
Auffrischen klebtechnischer Kenntnisse
}

Das Klebtechnische Zentrum des Fraunhofer-Instituts für Fertigungstechnik und Angewandte Materialforschung IFAM bot vom 14. bis 15. November 2012 erstmals den „Klebpraktiker-Refresher“ („European Adhesive Bonder-Refresher“/"EABRefresher“) als Fortbildung für DVS/EWF-Klebpraktiker in Bremen an.

$\mathrm{D}$ as neue Angebot des Fraunhofer IFAM bietet den Teilnehmern in kurzer Zeit die Möglichkeit, ihre klebtechnischen Kenntnisse aufzufrischen und sich über die Weiterentwicklungen der letzten Jahre sowie den aktuellen Stand der Klebpraktiker-Qualifizierung zu informieren.

Seit 1994 werden im Klebtechnischen Zentrum des Fraunhofer IFAM in Bremen international anerkannte klebtechnische Lehrgänge durchgeführt. Mittlerweile ist es nicht nur gelungen, das klebtechnische DVS/EWF-Personalqualifizierungssystem im deutschsprachigen Raum Europas zu etablieren, sondern auch in englischer Sprache international anzubieten. Außerdem konnte das Weiterbildungsangebot über die drei Lehrgangsstufen - „DVS/ EWF-Klebpraktiker“, „DVS/EWF-Klebfachkraft“ und „DVS/EWF-Klebfachingenieur“ - hinaus wie folgt erweitert werden:

Neben den inzwischen zweimal jährlich stattfindenden „Bremer Klebtagen“, die als allgemeine klebtechnische Fortbildung allen ehemaligen Kursteilnehmern und klebtechnisch Interessierten offenstehen, umfasst das Programm des Fraunhofer IFAM seit mehreren Jahren den so genann- ten „Klebfachkraft-Refresher“ („European Adhesive Specialist-Refresher“/"EAS-Refresher“), der allen ausgebildeten Klebfachkräften als Fortbildung dient, deren Weiterbildung schon länger zurückliegt.

Aufgrund der großen Nachfrage zum neuen „Klebpraktiker-Refresher 2012“ hat das Klebtechnische Zentrum nun beschlossen, ab 2013 im zweijährigen Turnus abwechselnd einen „Klebpraktiker-Refresher“ bzw. einen „Klebfachkraft-Refresher" anzubieten.

Weitere Infos: www.kleben-in-bremen.de

\section{Veranstaltungen}

\begin{tabular}{|l|l|l|l|}
\hline Termin & Ort & Thema & Veranstalter \\
\hline 14.-19.01.2013 & München & Bau 2013 & $\begin{array}{l}\text { Messe München GmbH } \\
\text { Tel: +49 (0)89 949 20720, www.bau-muenchen.com }\end{array}$ \\
\hline 26.-27.02.2013 & Frankfurt & $\begin{array}{l}\text { 12. Kolloquium: Gemeinsame } \\
\text { Forschung in der Klebtechnik }\end{array}$ & $\begin{array}{l}\text { DECHEMA e.V. } \\
\text { Tel.: +49 (0)69 7564-235, klebtechnik@dechema.de }\end{array}$ \\
\hline 12.-14.03.2013 & Paris & JEC & $\begin{array}{l}\text { JEC } \\
\text { Tel.: +33 158361500, www.jeccomposites.com }\end{array}$ \\
\hline 19.-21.03.2013 & Nürnberg & ECS European Coatings Show & $\begin{array}{l}\text { NürnbergMesse GmbH } \\
\text { Tel. +49 (0) 911 86 06-0, www.european-coatings-show.com/de }\end{array}$ \\
\hline 08.-12.04.2013 & Hannover & Industrial Supply & $\begin{array}{l}\text { Deutsche Messe } \\
\text { Tel.: +49 (0)511 - 89-31115, www.hannovermesse.de }\end{array}$ \\
\hline 16.-17.04.2013 & Stuttgart & $\begin{array}{l}\text { FTK - Fertigungstechnologie } \\
\text { Kleben 2013 }\end{array}$ & $\begin{array}{l}\text { Adhäsion Kleben \& Dichten } \\
\text { Tel: +49 (0)611 7878 283, www.adhaesion.com }\end{array}$ \\
\hline 16.-17.04.2013 & Stuttgart & Werkstoffe im Automobilbau & $\begin{array}{l}\text { ATZlive } \\
\text { Tel.: +49(0)611 7878-274, www.ATZlive.de }\end{array}$ \\
\hline Weitere Termine und Links zu den Veranstaltungen finden Sie auf unserer Website www.adhaesion.com
\end{tabular}

\section{Markttrends in der Klebtechnik}
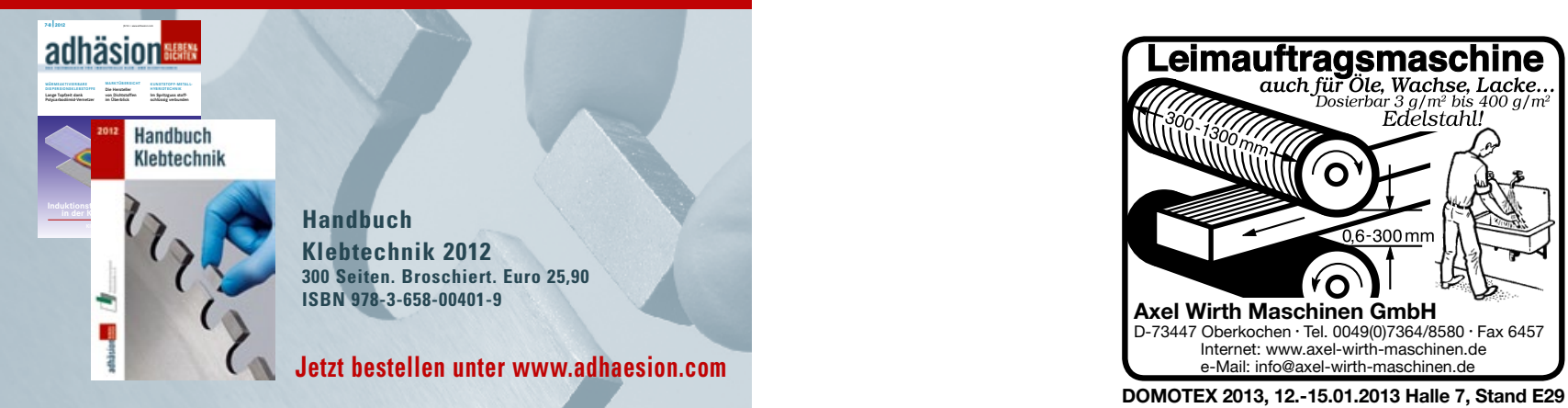